\title{
Corona-Krisenmanagement und Demokratie in Lateinamerika und der Karibik
}

\section{Claudia Zilla}

Am 30. Januar 2020 bewertete die Weltgesundheitsorganisation (WHO) der Vereinten Nationen die Ausbreitung des Coronavirus als Notlage internationaler Tragweite. Der erste Covid-19 Fall in Lateinamerika und der Karibik (LAK) wurde am 25. Februar in Brasilien ermittelt. Am 11. März erklärte die WHO Covid-19 zur Pandemie. Bis Dezember 2020 waren in LAK bereits 18,6 Prozent der Infektions- und 27,8 Prozent der Todesfälle weltweit registriert worden, obwohl in der Region lediglich 8,4 Prozent der Weltbevölkerung leben (CEPAL 2021a: 13). In diesen überdurchschnittlichen Covid-19 Werten drückt sich die ausgeprägte Verbundbarkeit des Subkontinents aus.

Verbreitete Armut und die größte soziale Ungleichheit der Welt gehören zu den Hauptaspekten dieser Verwundbarkeit. Privilegien sowie ihre Kehrseite, Exklusion und Benachteiligung, verteilen sich heterogen entlang verschiedener Kategorien wie Wirtschaftslage, Geschlecht, Alter, ethnisch-kultureller Identität, ${ }^{1}$ Behinderungen oder Wohnort. Zudem sind soziale Beziehungen durch niedriges Vertrauen und Diskriminierung geprägt. All diese Faktoren bedingen einen ungleichen Zugang zu Ressourcen und Dienstleistungen - auch in Bezug auf die segmentierten Gesundheitssysteme der Region. Aufgrund dieser Kontextbedingungen entstehen ungleiche Chancen, sich mit Covid-19 anzustecken, daran zu erkranken oder zu sterben. Die kulturellen und sozio-ökonomischen Asymmetrien führen zudem dazu, dass verschiedene Gesellschaftsgruppen von den Nebenfolgen der politischen Maßnahmen zur Seuchenbekämpfung unterschiedlich (negativ) betroffen sind. Daraus ergibt sich ein intersektionaler Teufelskreis der Verstetigung und Verschärfung struktureller Ungleichheiten, der nur mit massivem, ausgleichendem, staatlichem Eingriff gebrochen werden kann. Staatsintervention erfordert aber politischen Willen, Finanzressourcen und Staatskapazität und setzt zuallererst voraus, dass die Situation als zu bewältigendes Problem bewertet wird.

1 Laut CEPAL (2021a: 18) gehören in LAK 58 Millionen Menschen indigenen Völkern an, während 134 Millionen Menschen afrikanischer Abstammung sind. 
Ähnlich verhält es sich mit dem Coronavirus. Als im Jahr 2020 die Pandemie ausbrach, folgten nicht alle Staatoberhäupter der Welt der alarmierenden Einschätzung der WHO (sofort). Auch in LAK gingen die Meinungen auf höchster Ebene bezüglich der Fragen, wie ernst der sanitäre Fall und inwiefern außerordentliches Regierungshandeln vonnöten sei, (lange) auseinander. Heterogene Ansichten trennten die Regierungen nicht nur verschiedener Länder, sondern bisweilen auch die Exekutiven auf verschiedenen Staatsebenen in ein und demselben Land. In diesem Zusammenhang wirkt sich Föderalismus ambivalent aus: Er kann einerseits die Koordinierung eines einheitlichen und wirksamen Krisenmanagements erschweren und Ungleichheit zwischen Staatskörperschaften erzeugen (wie in Argentinien). Andererseits kann er in Krisensituationen als Gegengewicht zur nationalen Exekutive fungieren und der Tendenz zu deren Kompetenzüberschreitung oder Untätigkeit (wie beispielsweise in Brasilien) entgegenwirken.

Trotz der unterschiedlichen Bewertungen bildet LAK die Weltregion, in der Regierungen die meisten, strengsten und längsten Lockdowns bzw. Ausgangssperren verhängten. Diese Maßnahmen stellten politischrechtliche Instrumente zur Pandemie-Eindämmung dar, bedeuteten aber zugleich eine Einschränkung sensibler Rechte und Freiheiten und hatten für viele verheerende sozio-ökonomische Folgen. Die meisten Regierungen der Region waren nicht gleichgültig gegenüber diesen Nebenwirkungen und trafen eine Reihe finanzieller wie nicht-finanzieller kompensatorischer Maßnahmen, um diese abzufedern. In LAK erfolgte und erfolgt das politische Corona-Krisenmanagement im Rahmen fragiler Demokratien, die durch einen von Armut und sozialer Ungleichheit geprägten Kontext extrem unter Druck stehen. Dabei unterscheiden sich die Staaten der Region in ihrem Grad demokratischer Qualität - und einige politische Regime wie Kuba und Venezuela, aber auch mittlerweile Nicaragua sogar als autoritär eingestuft werden müssen.

$\mathrm{Zu}$ den Gesamtimplikationen der Corona-Krise gehören also - neben den direkten Effekten der Covid-19-Pandemie - auch die Folgewirkungen des nationalen politischen Pandemie-Managements sowie weiterer externer Faktoren (Zilla 2020a). Im vorliegenden Beitrag nehme ich jedoch eine dreifache Fokussierung vor: Im Mittelpunkt der Analyse stehen die negativen Auswirkungen des politischen Umgangs mit der Corona-Krise auf die Qualität von Demokratie. Dabei verstehe ich den „politischen Umgang mit der Corona-Krise“ im weitesten Sinne, denn ich berücksichtige politische Entscheidungen und Maßnahmen, die unter Verweis auf die Covid-19-Pandemie begründet wurden - und zwar ungeachtet dessen, ob sie tatsächlich dem vermeintlichen Zweck dienen. Aus dieser Perspektive 
ergibt sich ein problemorientierter Ansatz. Nicht behandelt werden hier die unmittelbaren Effekte der Covid-19-Pandemie im Allgemeinen sowie die mittelbaren Auswirkungen des politischen Krisenmanagements auf den sozialen und ökonomischen Kontext von Demokratie. Diese sind zwar nicht minder relevant für die Qualität und die Funktionsweise des politischen Regimes, bilden aber den Gegenstand anderer Beiträge in diesem Buch (vgl. die Beiträge von Burchardt, Carosio, Kim, Krawinkel, Weller oder Vestena in diesem Band).

Der vorliegende Beitrag ist wie folgt strukturiert: Im ersten Abschnitt erläutere ich das minimale, normativ-prozedurale Verständnis von Demokratie, das der folgenden kritischen Analyse zugrunde liegt. Sodann diskutiere ich im zweiten Abschnitt vier Entwicklungen in Lateinamerika, die im Zusammenhang mit dem politischen Corona-Krisenmanagement stehen und die Qualität von Demokratie unter Druck setzen: (1) Korruption und Privilegien, (2) Einschränkung des freien Informationszuganges, (3) Verschiebung von Wahlen und Restriktionen des Versammlungs- und Demonstrationsrechts und (4) Freiheitsentzug, sanitärer Notstand und Ausnahmezustand. Im dritten abschließenden Abschnitt ziehe ich ein Fazit und gewichte dabei die sozio-ökonomischen und politischen Folgewirkungen des Corona-Krisenmanagements.

\section{Demokratie als Freiheit und Gleichheit}

Ein normativ-prozedurales Konzept von Demokratie (Urbinati/Warren 2008) geht von Prinzipien aus, auf die sich Verfahren stützen. Die Prinzipien bilden die normative Grundlage; die Verfahren ermöglichen deren prozedurale Umsetzung. Minimal bleibt ein Demokratiebegriff dann, wenn seine Definition wenige, in erster Linie bürgerrechtliche und politische Dimensionen umfasst, während weitere, etwa wirtschaftliche Aspekte außen vor bleiben. Dies bedeutet nicht, Demokratie von anderen, substantiellen oder materiellen Variablen vollkommen unabhängig zu verstehen. Es geht vielmehr um die Unterscheidung zwischen Demokratie und Kontext, zwischen dem politischen Regime und jenen sozio-ökonomischen Faktoren, die es stärken oder gefährden.

Die beschriebenen Eigenschaften treffen auf das hier verwendete Demokratiekonzept zu, das normativ auf der Anerkennung zweier Prinzipien beruht: Freiheit und Gleichheit. Freiheit kann in einem liberalen (negativen) Sinn als Nicht-Herrschaft und in einem republikanischen (positiven) Sinn als Selbstregierung verstanden werden (Urbinati 2012). Im ersteren, negativen Sinn bedeutet Freiheit die Abwesenheit willkürlicher Einmischung 
bzw. Machtkontrolle, das heißt der Schutz von Rechten und Freiheiten vor Dritten. Sie impliziert auch Rechtsstaatlichkeit sowie Begrenzung der Autorität und zwar nicht nur mit Bezug auf eine herrschende Einzelperson, sondern auch auf eine Volksmehrheit, deren Wille ebenfalls durch das Recht und den Schutz von Minderheiten eingeschränkt ist. Im zweiten, positiven Sinn bedeutet Freiheit Autonomie: die Befolgung von Gesetzen, die man selbst gemacht hat. Damit ist der Anspruch gemeint, dass alle Mitglieder einer politischen Gemeinschaft, deren Interessen von kollektiven Entscheidungen betroffen sind, ein Mitspracherecht haben (Rueschemeyer 2011: 819). Die Begriffe Konstitutionalismus und Volkssouveränität spielen in der Regel auf diese beiden Bedeutungen von Freiheit als Nicht-Herrschaft und Selbstregierung an.

Gleichheit wiederum bedeutet Gleichheit zwischen den Bürger:innen. Dieses Prinzip gründet sich auf das moralische Urteil, dass alle Menschen gleiche Würde besitzen und gleiche Anerkennung verdienen (Dahl 2006: 4; Rueschemeyer 2011: 819). Daraus leiten sich die bürgerliche Gleichheit vor dem Gesetz (Konstitutionalismus) und die politische Gleichheit in dem Sinne ab, dass alle Bürger:innen gleiche Einflussmöglichkeiten auf die kollektive Entscheidungsfindung eines politischen Gemeinwesens haben sollen (Volkssouveränität) (Rueschemeyer 2011: 818).

Nach diesem Verständnis sind die Prinzipien Freiheit und Gleichheit der Demokratie gleichermaßen intrinsisch. Gemäß einem minimalen Konzept von Demokratie gilt dies jedoch nicht für sozio-ökonomische Gleichheit. Verfechter:innen substantieller Demokratiebegriffe erachten Demokratie hingegen als Gesamtverfassung, die nicht nur die Sphäre der Politik, sondern auch der Wirtschaft und/oder der Gesellschaft umfasst. Beispielsweise beinhaltet der Begriff „soziale Demokratie“ (Meyer 2006 und 2011) sozio-ökonomische Gleichheit als weitere definitorische Kategorie von Demokratie. Zweifelsohne stellt es eine demokratische Herausforderung dar, bürgerliche und politische Gleichheit unter Bedingungen signifikanter sozio-ökonomischer Ungleichheiten zu gewährleisten. Wenn sozio-ökonomische Ungleichheiten keinerlei Ausgleich erfahren, können bürgerliche und politische Gleichheit nicht voll verwirklicht werden (Rueschemeyer 2011: 819). Ähnlich verhält es sich im Falle angeborener Begabungen und angeeigneter Fähigkeiten. Beispielsweise werden sich Menschen mit heterogenen rhetorischen und organisatorischen Kompetenzen auch in der Fähigkeit unterscheiden, ihre politischen Präferenzen zu artikulieren und durchzusetzen. Sozio-ökonomische Ungleichheit sowie die Ungleichheit in den Kompetenzen führen also zu ungleichen Chancen, politischen Einfluss auszuüben. 
Um die Demokratie als politisches Regime vor diesen Ungleichheiten zu schützen, das heißt um sie unter diesen erschwerten Bedingungen zu einem gewissen Grad gewährleisten zu können, ist politische Regulierung sowohl der Demokratie selbst als auch des Kontextes notwendig. Im ersten Fall geht es darum, das politische System derart zu gestalten, dass es gegenüber dem sozialen und Wirtschaftssystem immunisiert wird bzw. dass sich die sozialen und ökonomischen Machtunterschiede nicht oder zumindest so wenig wie möglich in politische Machtunterschiede übersetzen. Diesem Ziel diente etwa die Abschaffung des Zensuswahlrechts ${ }^{2}$ oder die Einführung von Frauenquoten für die Legislative. Im zweiten Fall geht es darum, durch gezielte Maßnahmen für den Abbau sozio-ökonomischer Asymmetrien außerhalb des politischen Systems zu sorgen. Dies verfolgen beispielsweise Regierungen, wenn sie progressive Steuersysteme einführen oder eine Frauenquote für Unternehmensvorstände beschließen. Sowohl bei der Immunisierung des politischen Systems gegenüber seinem Kontext durch Demokratiegestaltung als auch beim politischen Eingriff in die Beschaffenheit des sozio-ökonomischen Kontextes handelt es sich um Inklusionsmaßnahmen, die darauf ausgerichtet sind, den Grad an demokratischer Freiheit und Gleichheit hoch zu halten oder zu erhöhen.

\section{Freibeit und Gleichheit unter Druck}

Im Rahmen des Corona-Krisenmanagements in LAK waren viele Maßnahmen darauf ausgerichtet, nicht nur die Menschen vor Covid-19, sondern auch demokratische Prinzipien und Verfahren vor sanitären Restriktionen zu schützen. Es gab bedeutende Bemühungen, diese Trade-Off-Beziehung mit Bedacht zu behandeln. Zahlreich sind aber auch die Fälle, in denen das Spannungsverhältnis zwischen Gesundheitsschutz und Schutz von Rechten und Freiheiten erst durch nicht-demokratisches bzw. demokratieschädliches Regierungshandeln entstand. Ich konzentriere mich nun auf diesen letzten Falltypus, der ausgehend vom oben erläuterten Demokratiebegriff deutliche Kritik verdient.

2 Das Zensuswahlrecht stellt ein ungleiches Wahlrecht dar, nach dem nur diejenigen wählen dürfen, die Finanzmittel (Grundbesitz, Vermögen etc.) nachweisen können. 


\section{Korruption und Privilegien}

Ein Vehikel zur (zumeist ökonomisch bedingten) Verzerrung des bürgerlichen Gleichheitspostulats stellt politische Korruption dar, denn sie mündet in einen ungleichen Zugang zu öffentlichen materiellen wie immateriellen Ressourcen abhängig von Finanzmitteln und „Beziehungen“. Korruption stellt ein Fehlverhalten dar, in dessen Mittelpunkt der Missbrauch eines öffentlichen Amtes durch Normverstoß und Inkaufnahme der Schädigung von Kollektivinteressen steht. Dieser erfolgt zum Zwecke der Mehrung privaten Nutzens oder der Vorteilserlangung für Dritte und wird in der Regel geheim gehalten (Schmidt 2004: 390). Politische Korruption zieht die Fehlallokation öffentlicher Ressourcen nach sich und unterminiert das Vertrauen der Bürger:innen in die Amts- und Mandatsträger:innen sowie in die Institutionen des Staates.

In LAK stellt Korruption seit jeher ein strukturelles Problem dar, denn sie kommt nicht einfach sporadisch in Einzelsituationen vor, sondern prägt vielmehr die Funktionsweise von Politik und die Staatsstrukturen. $\mathrm{Zu}$ diesem komplexen Phänomen tragen - neben der hohen Straflosigkeit - die sozio-ökonomischen Asymmetrien bei. Umgekehrt behindert strukturelle Korruption sozialgerechte und nachhaltige Entwicklung. Zwar wurde in der letzten Dekade eine Reihe von politischen wie juristischen Maßnahmen zur Korruptionsbekämpfung wie etwa in Chile und Brasilien eingeführt (Wesche/Zilla 2017). Das Problem besteht jedoch weiterhin, wie die intensive Berichtserstattung über Korruptionsskandale in den regionalen Medien offenlegt.

In vielen LAK-Ländern wurden seit Ausbruch der Pandemie Unregelmäßigkeiten und Korruptionsfälle in verschiedenen Ministerien und Behörden bei der Beschaffung von Beatmungsgeräten, Covid-19 Tests, Leichensäcken, Krankenwagen und verschiedenen Biosicherheitsmaterialien gemeldet, die mitten in der Corona-Krise dringend benötigt werden. Zahlreiche Beamte und lokale Geschäftsleute werden beschuldigt, die Krise für ihre persönliche Bereicherung ausgenutzt zu haben, indem sie Verträge über überteuerte Grundversorgungsgüter miteinander schlossen (TNYT 2020). Die Preisdifferenz wird in der Regel zwischen den an der Transaktion beteiligten Parteien aufgeteilt oder lediglich von der politischen Seite einkassiert als „Gegenleistung “ für die Erteilung des öffentlichen Auftrags an die private Seite. Einige der unter Bedingungen der Korruption gelieferten Produkte und Geräte waren fehlerhaft und konnten entweder nicht eingesetzt werden oder die Menschen vor der Pandemie nicht effektiv schützen. Ein Bespiel hierfür bilden die 324 mutmaßlich (um sechs Millionen US-Dollar) überteuerten Beatmusgeräte, die die bolivianische 
Regierung aus China kaufte (Infobae 2020). In acht der insgesamt neun bolivianischen Departements, in denen sie Einsatz fanden, beklagte das sanitäre Personal, dass diese entweder schlecht funktionierten oder für die Linderung der medizinischen Notlage nicht geeignet waren.

Korruptes Fehlverhalten wurde auch mehrfach bei der Verteilung der Impfstoffe festgestellt. So entstanden im Kontext der Corona-Krise die Begriffe „vacunas VIP“ und „vacunagate“: Akteur:innen mit politischer Autorität setzten sich selbst sowie eigene „Freund:innen“ auf geheime Listen, wodurch sie privilegierten Zugang zur Covid-19-Impfung erhielten. Diese Priorisierung befolgt keine allgemeinen Regeln, die sich auf gesundheitliche Gesichtspunkte stützen, sondern basiert lediglich auf der Bevorzugung der eigenen Person sowie von Verwandten, Bekannten und berühmten Persönlichkeiten bei der Verabreichung des Impfstoffes.

Zwischen dem Ausbruch der Corona-Pandemie in LAK Anfang 2020 und März 2021 mussten über 20 Gesundheitsminister:innen ihr Amt aufgeben, die meisten von ihnen inmitten von Korruptionsvorwürfen im Zusammenhang mit dem Corona-Krisenmanagement. Hierzu zählen etwa die Ressortleitungen in Argentinien (Ginés González), Bolivien (Marcelo Navajas), der Dominikanischen Republik (Plutarco Arias), Ecuador (Catalina Andramuño, Juan Carlos Zevallos und Rodolfo Farfán), Gautemala (Hugo Monroy sowie Vizeminister Danilo Sandoval Flores, Rodolfo Galdámez und Héctor Marroquín und der Chef für Epidemiologie Manuel Sagastume) Paraguay (Julio Mazzoleni) und Peru (Pilar Mazzetti) (NODAL 2021; France24 2021a; DW 2020a; El Nacional 2021; France21 2021b; Listin Diario 2021; Coprofam 2021).

\section{Einschränkung des freien Informationszugangs}

Die Korruptionsbekämpfung, die Kontrolle politischer Macht durch das souveräne Volk sowie die damit verbundene Rechenschaft von Regierenden gegenüber den Regierten setzt die freie Zirkulation von Information voraus. Diese ist in der Meinungs- und Pressefreiheit sowie in dem Recht auf öffentliche Information durch den Staat verankert. Demnach hat jede Person das Recht, unentgeltlich Informationen (Auskunft wie Akteneinsicht) anzufordern, die von öffentlichen Stellen erzeugt oder verwaltet werden oder sich in deren Besitz befinden. Staatliche Stellen sind wiederum dazu verpflichtet, die Informationen herauszugeben, ohne dass die beantragende Person ein Interesse nachweisen oder ihre Verwendung rechtfertigen muss. Seit 2002 haben immer mehr LAK-Staaten Transparenzgesetze (in Deutschland Informationsfreiheitsgesetz genannt) erlassen, 
die den voraussetzungslosen Anspruch auf Zugang zu amtlichen Informationen bei Behörden rechtlich herstellen. Diesem Trend blieben bisher lediglich Bolivien, Costa Rica, Dominica, Granada, Haiti, Kuba, Santa Lucia, Surinam und Venezuela fern (CEPAL 2021b).

Im Kontext der Covid-19 Pandemie erlaubt die Informationsfreiheit den Bürger:innen, die Performanz bzw. das Handeln der Regierung beim Krisenmanagement zu bewerten und zu überwachen. Dieser Beobachtung ausgesetzt haben einige Regierungen versucht, ihre Leistungen durch gezielte „Informationspolitik“ zu verschleiern oder zu beschönigen. Derartige Absichten und Maßnahmen werden meist durch zivilgesellschaftliche Organisationen oder investigative Journalisti:nnen aufgedeckt, die eine wichtige Aufgabe bei der gesellschaftlichen Kontrolle staatlicher Politik (social accountability) ausüben. Aber auch eine funktionierende Gewaltenteilung sorgt für Transparenzschutz (borizontal accountability).

In Chile musste im Juni 2020 der Gesundheitsminister Jaime Mañalich zurücktreten, als bekannt wurde, dass die Statistikabteilung seines Ressorts den Bürger:innen (weniger) und der WHO (mehr) unterschiedliche Daten über die Covid-19 bedingten Todesfälle im Lande lieferte (DW 2020b). Einmal publik rechtfertigten Amtsträger:innen diese Abweichungen häufig mit Verweis auf die Anwendung verschiedener Berechnungsmethoden. Im Falle Brasiliens wiesen Judikative und Legislative die Exekutive in die Schranken: Im Juni 2020 hatte Präsident Jair Bolsonaro entschieden, nicht mehr die (extrem hohe) Gesamtzahl von Covid-19-Infektionen zu veröffentlichen, sondern nur die täglich neu registrierten. Dies musste er allerdings revidieren, nachdem ein Richter des Obersten Bundesgerichtshofs auf Antrag von Oppositionsparteien eine einstweilige Verfügung erließ. In der Zwischenzeit hatte das brasilianische Parlament bereits seine Absicht bekundet, die Gesamtzahl der Krankheitsfälle zu verfolgen und zu veröffentlichen (Zilla 2020b: 5).

In Venezuela wurden Journalist:innen, die die Infektionszahlen unterschiedlicher Behörden in Frage stellten und selbst recherchierte Daten zur Verfügung stellen festgenommen (BBC 2021): Diese Erfahrung musste etwa Darwinson Rojas durchmachen, der zwölf Tage in Haft blieb, weil er die Infektionsdaten verschiedener öffentlicher Stellen zusammengeführt und auf Twitter veröffentlicht hatte. Dabei handelt es sich nicht um einen Einzelfall. Bereits im April 2020 berichtete das Nationale Journalistenkollegium über die Verhaftung von 18 Journalist:innen. Von willkürlichen Festnahmen waren in Venezuela auch Mitglieder des medizinischen und sanitären Personals betroffen, als sie Defizite des staatlichen Gesundheitssystems in der Öffentlichkeit anprangerten. Diese Fälle staatlicher Repres- 
sion stiften Angst in der Bevölkerung und fördern damit auch die Selbstzensur.

Die Regierung Guatemalas schränkte ebenfalls den Zugang zur öffentlichen Information stark ein und ging gegen Journalist:innen vor, die offen über die Pandemieentwicklung und das Krisenmanagement der Regierung informierten, wie eine gemeinsame Erklärung vom Februar 2021 von Human Rights Watch (HRW 2021a) und dem Komitee für den Schutz von Journalist:innen (CPJ) beklagte (s. Krawinkel zu Nicaragua in diesem Buch).

\section{Verschiebung von Wablen und Restriktionen des Versammlungs- und Demonstrationsrechts}

Freie Wahlen sowie der Schutz des Versammlungs- und Demonstrationsrechts gehören zu den wichtigsten Institutionen politischer Partizipation. Demokratische Partizipation soll nicht nur unter freiheitlichen, sondern auch gleichen Bedingungen stattfinden.

Seit dem Ausbruch der Pandemie und mit Verweis auf diese wurden in LAK über ein Dutzend der 16 Wahlprozesse verschoben, die für das Jahr 2020 geplant waren (IDEA 2021). Hierzu zählen Urnengänge auf verschiedenen Staatsebenen in Argentinien, Bolivien, Brasilien, Chile, der Dominikanischen Republik, Kolumbien, Mexiko Paraguay, Peru und Uruguay. Je nach Land und Rechtslage wurde die Anpassung des elektoralen Zeitplans durch die Wahlbehörde oder ein Gesetz der Legislative entschieden. Die neue Terminierung wurde mehrheitlich eingehalten. Verschobene Wahlen wurden unter Anwendung eines sanitären Corona-Protokolls durchgeführt. Je nach Abstand zwischen dem alten und dem neuen Wahltermin, entsteht durch die Verschiebung eine variable Ungleichheit bei der Mandats- bzw. Amtsdauer der Volksvertreter:innen.

Aus normativ-demokratischer Perspektive erscheint jedoch die zeitliche Verschiebung von Wahlen weniger problematisch als die großen Einschränkungen, die Eindämmungsmaßnahmen für politische Aktivitäten im Vorfeld der Wahlen bedeuten. Denn Wahlkampf setzt die freie Ausübung des Versammlungs- und Demonstrationsrechts voraus. Wenn ein Wahlkampf nur unter hohen Auflagen stattfinden kann, wird der Vorteil für die Regierungsparteien (üblicherweise sichtbarer und reicher an Ressourcen) umso größer.

Laut der UN-Wirtschaftskommission für Lateinamerika und die Karibik (CEPAL 2021c) führten so gut wie alle Staaten der Region (96 Prozent) Maßnahmen ein, die Massenevents und die Nutzung öffentlicher Räume 
für politische Veranstaltungen einschränkten. In vielen Fällen erfolgten diese Einschränkungen im Zusammenhang mit der Verhängung des sanitären Notstands bzw. des Ausnahmezustands aufgrund einer Katastrophe.

\section{Freibeitsentzug, sanitärer Notstand und Ausnabmezustand}

Außerordentliches Regierungshandeln in Krisensituationen erfordert häufig erweiterte Kompetenzen und erhöhte Ressourcen. Damit die Exekutive Sonderbefugnisse übernehmen und andere Instanzen mit Mitspracherecht im Regelfall zugunsten der Zügigkeit vom Entscheidungsprozess im Krisenfall ausschließen kann, wird oft per Dekret (der Exekutive) oder Gesetz (der Legislative) ein hierfür notwendiger Rechtsrahmen gesetzt. Diese Aufgabe erfüllt die Verhängung des Notstands bzw. Ausnahmezustands. Anlass dazu gibt eine Situation, in der öffentliche Sicherheit und Ordnung als schwerwiegend gestört oder gefährdet erachtet werden. Es handelt sich also um eine Notsituation, in der die Existenz des Staates und die Erfüllung der staatlichen Grundaufgaben sowie die Integrität der Bevölkerung durch eine interne oder externe Instanz als akut bedroht angesehen werden. Durch die Verhängung des Notstands bzw. Ausnahmezustands wird die Exekutive unter anderem dazu ermächtigt, gewisse Freiheiten und Rechte der Bürger:innen einzuschränken. Zwar sind graduelle, temporäre, notwendige und verhältnismäßige Restriktionen ziviler und politischer Rechte nicht per se verfassungswidrig, jedoch rechtlich wie demokratisch extrem voraussetzungsvoll.

Die meisten Regierungen der Region haben im Kontext des CoronaKrisenmanagements die ökonomischen Aktivitäten heruntergefahren, die Grenzen geschlossen, obligatorische soziale Isolierung verordnet, Lockdowns und Ausgangssperren verhängt. Diesen Entscheidungen ging fast immer die Ausrufung des sanitären Notstands voraus. Die Restriktionen des Demonstrations- und Versammlungsrechtes sowie der Freizügigkeit ab März 2020 wurden in einigen Ländern durch Protestwellen unterbrochen, so etwa in Bolivien und Chile. In anderen Staaten gaben sie Anlass zu repressiven Maßnahmen gegen die Bevölkerung.

Ermutigt durch den drakonischen Diskurs von Präsident Nayib Bukele nahm die Polizei in El Salvador bereits seit März 2020 hunderte von Personen willkürlich fest und internierte viele von ihnen in überfüllte und unhygienische Quarantänezentren. Dabei gab es sogar Todesfälle aufgrund fehlender angemessener medizinischer Versorgung (HRW 2020). Einige Personen wurden auf den Straßen festgehalten, weil sie (im Freien) keine Masken trugen, obwohl das obligatorische Tragen eines Mund- 
schutzes nicht verordnet war und es aufgrund eines Engpasses im Land keine Masken zu kaufen gab. Der Umgang der Sicherheitskräfte mit den Bürger:innen im Kontext der Pandemie war geprägt von rechtswidrigen Maßnahmen, die in ihrer Form, ihrem Anlass oder mit Bezug auf die Personengruppe durch das Dekret des Präsidenten nicht gedeckt waren. Allerdings war Bukele bereits vor der Pandemie durch seinen populistischen und aggressiven Regierungsstiel aufgefallen.

Für missbräuchliche freiheitsentziehende Maßnahmen waren nicht nur Präsidenten verantwortlich, sondern auch einige Exekutive unterer Staatsebenen. So haben Menschenrechtsorganisationen das Corona-Krisenmanagement von Gildo Insfrán, seit 1995 (sic) Gouverneur von Formosa, einer Provinz im föderalen Argentinien, kritisiert. Die Behörden in Formosa zwangen seit April 2020 mehr als 24.000 Menschen zum Verbleib in so genannten Isolations- und Quarantänezentren (HRW 2021b). Einige Betroffene wurden sogar länger als die von der WHO empfohlenen 14 Tage in diesen Zentren eingesperrt. In vielen Fällen erfolgte dies unter Umständen, die als willkürliche Inhaftierung zu bewerten sind. Die Behörden Formosas haben einige Personen, die positiv auf Covid-19 getestet waren, neben anderen festgehalten, die negativ getestet waren oder noch auf ihre Testergebnisse warteten. Die Überbelegung und die unhygienischen Bedingungen in den Zentren haben die soziale Distanzierung verhindert oder zumindest erschwert. Doch nicht erst seit Ausbruch der Pandemie stehen Entscheidungen und Maßnahmen des Gouverneurs von Formosa im Spannungsverhältnis mit demokratischen Prinzipien. Sein Regierungsstil weist seit jeher starke autoritäre und rassistische Züge auf. Von der Repression durch den Sicherheitsapparat der Provinz sind Teile der Bevölkerung wie der politischen Opposition betroffen.

Ein extremes Beispiel in diesem Zusammenhang bildet Venezuela, wo mit Bezug auf die Pandemie der Ausnahmezustand erneut ausgerufen wurde. Die Pandemie lieferte jedoch nur den Vorwand für die Fortsetzung einer chronisch autoritären Situation, denn zwischen 1999 und 2019 wurde der Ausnahmezustand in Venezuela mit verschiedenen Begründungen per Dekret mindestens $29 \mathrm{Mal}$ auf lokaler, regionaler oder nationaler Ebene verhängt. Seit 2015 intensivierte Präsident Nicolás Maduro diese Praxis, ab 2020 mit Verweis auf die Corona-Krise. Das Resultat ist eine so gut wie lückenlöse Verkettung von Ausnahmezustands- und Verlängerungsdekreten, die aus heterogener wie willkürlicher Weise begründet werden (Zilla/Keseberg 2019). Vor diesem Hintergrund rechtfertigt weder die Pandemie die Verhängung des Ausnahmezustands noch dieser die massiven Menschenrechtsverletzung unter der Regierung von Nicolás Maduro. 


\section{Lateinamerika und die Karibik - weniger demokratisch durch „Corona“?}

Krisensituationen bedingen ein "schmales“, die Partizipation und Kontrolle politischer Macht abschwächendes Präferenzmuster: Das Erzielen von Wirkung (Effektivität) wird in der Regel gegenüber Wirtschaftlichkeit (Effizienz) priorisiert; schnelle Reaktionen und Entschlossenheit (Dezisionismus) erscheinen dringender als Transparenz, Inklusion, die Berücksichtigung von Alternativen und Rechenschaftsablegung (accountability) im Entscheidungsprozess. Vor diesem Hintergrund ist es nicht verwunderlich, dass in den präsidentiellen Systemen des Subkontinents die Maßnahmen im Rahmen des Corona-Krisenmanagements nicht nur, aber vor allem, von den jeweiligen Exekutiven getroffen wurden.

Viele LAK-Regierungen, die den sanitären Not- bzw. Ausnahmezustand verhängten, hoben im Dienste der Zügigkeit und funktionaler Fokussierung zugleich Regulierungen auf, die für das öffentliche Auftragswesen gelten, sowie das Recht auf Information und auf Auskunft über Vorgänge von Behörden. Zudem suspendierten zahlreiche Parlamente die Präsenzsitzungen; bei den Gerichten wurden außerordentliche Ferien dekretiert. Dadurch verringerten sich die gesellschaftlichen und politischen Kontrollmöglichkeiten über die politische Macht und das Regierungshandeln. Gleichzeitig entsteht ein Nährboden für Korruption.

Dennoch hat die Corona-Pandemie LAK weder undemokratischer gemacht, noch irgendeinen Staat der Region in den Autoritarismus fallen lassen. Es gibt auch keine Evidenz dafür, das populistische Tendenzen sich intensiviert hätten (Lang/Zilla 2020). Dies ist der Fall aus zweierlei Gründen: Zum einen sind in der Region demokratische Defizite, die sowohl die bürgerliche und politische Gleichheit als auch die Machtkontrolle und die politische Partizipationschancen betreffen, nicht neu. Es lässt sich keine lineare Konsolidierung oder Demokratisierung der Demokratie beobachten, die gestoppt oder umgekehrt wurde. Es besteht vielmehr eine dynamische Gleichzeitigkeit des Ungleichzeitigen - je nach Land, politischem Bereich und Zeitraum. Zum anderen ist der Corona-Virus kein Akteur, der politisches Handeln verantwortet (Zillla 2020c). Die Pandemie prägt vielmehr (zusammen mit anderen Faktoren) den Kontext, in den das politische Regime eingebettet ist. Die pandemiebedingten Veränderungen des Kontextes schränken den Spielraum für bestimmte politische Handlungen ein, schaffen oder erweitern aber auch Gelegenheitsstrukturen für andere wie beispielsweise autoritäre Maßnahmen oder korrupte Praktiken. Meistens wirkt sie als Brennglas, das die bereits bestehenden strukturellen Probleme deutlicher erscheinen lässt. 
Die Effekte der Pandemie und die Nebenfolgen des politischen Corona-Krisenmanagements hinterlassen den LAK-Demokratien ein schweres Erbe. CEPAL (2021b: 16) prognostiziert zum Ende 2020 einen ökonomischen Einbruch in der Region von -7,7 Prozent. Die Staatsverschuldung ist stark gestiegen. Die Zahlen zur extremen Armut (12,5 Prozent) und zur Armut (33,7 Prozent) werden Rekordwerte erreichen. Der Gini-Koeffizient zur Messung von Einkommensungleichheit dürfte durchschnittlich um 2,9 Prozent ansteigen, was eine empfindliche Verschlechterung der Situation bedeutet. Von diesen negativen Entwicklungen sind Arme, Frauen, Mitglieder der LGBTQI-Community sowie indigene und afrikanisch-stämmige Bevölkerungsgruppen in besonderem Maße betroffen. So dürften die sozio-ökonomischen Nebenfolgen der Pandemie und des politischen Corona-Krisenmanagements eine ungleich größere Herausforderung für die LAK-Demokratien darstellen, als die eingeführten Restriktionen im politischen System selbst. Daher erfordert die Stärkung der Demokratien in Lateinamerika und der Karibik nicht nur die (volle) Wiederherstellung von Freiheiten und Rechten, sondern auch eine umfassende, soziale Ungleichheiten reduzierende, Intervention in ihre Kontexte.

\section{Literaturverzeichnis}

BBC (2021): Coronavirus en Venezuela: los periodistas y médicos detenidos en el país en medio de la pandemia, |https://www.bbc.com/mundo/noticias-america-latina-524 50803], letzter Aufruf 08.09.2021.

CEPAL (2020): Panorama Social de América Latina y el Caribe 2020, Santiago de Chile: CEPAL.

CEPAL (2021b): Observatorio del Principio 10 en América Latina y el Caribe [https://o bservatoriop10.cepal.org/es/recursos/america-latina-caribe-paises-que-cuentan-ley -acceso-la-informacion-publica-ano], letzter Aufruf 08.09.2021.

CEPAL (2021c): Observatorio COVID-19 en América Latina y el Caribe: acciones por pais, (laufend aktualisierte Datenbank) [https://www.cepal.org/es/temas/covid-19 ], letzter Aufruf 08.09.2021.

Coprofam (2021): De héroe a villano: degastado por casos de corrupción renuncia el ministro de Salud. [https://coprofam.org/2021/03/06/de-heroe-a-villano-degas tado-por-casos-de-corrupcion-renuncia-el-ministro-de-salud/], letzter Aufruf 08.09.2021.

Dahl, Robert (2006): On Political Equality, New Haven \& London: Yale University Press. 
DW (2020a): Arrestan a ministro de salud de Bolivia y ordenan auditoría por escándalo respiradores. [https://www.dw.com/es/arrestan-a-ministro-de-salud-de-bolivia-y -ordenan-auditor\%C3\%ADa-por-esc\%C3\%A1 ndalo-respiradores/a-53520623], letzter Aufruf 08.09.2021.

DW (2020b): Renuncia el ministro de Salud en Chile en medio de la polémica. [https:// www.dw.com/es/renuncia-ministro-de-salud-de-chile-en-medio-de-pol\%C3\%A9 mica/a-53798158], letzter Aufruf 08.09.2021.

El Nacional (2021): En 6 meses de gobierno, Abinader destituye 1 ministro y otro le renuncia. [https://elnacional.com.do/en-6-meses-de-gobierno-abinader-destituye-1 -ministro-y-otro-le-renuncia/], letzter Aufruf 08.09.2021.

France24 (2021a): Argentina: renuncia ministro de Salud, Ginés González, por el escándalo "vacunación VIP". [https://www.france24.com/es/am\%C3\%A9rica-la tina/20210219-argentina-alberto-fernandez-renuncia-ministro-salud-vacunacion-c ovid19], letzter Aufruf 08.09.2021.

France24 (2021b): Ecuador: renuncia el tercer ministro de Salud desde inicio de la pandemia, [https:/www.france24.com/es/am\%C3\%A9rica-latina/20210319-farfa n-ecuador-salud-ministro-renuncia-polemica], letzter Aufruf 08.09.2021.

HRW (2020): El Salvador: Abusos Policiales en la Respuesta a la Covid-19: Detenciones arbitrarias, condiciones riesgosas en detención y cuarentena. [https://www.hrw.org/ es/news/2020/04/15/el-salvador-abusos-policiales-en-la-respuesta-la-covid-19], letzter Aufruf 08.09.2021.

HRW (2021a): Guatemala: Ataques a la libertad de prensa. [https://www.hrw.org/ es/news/2021/02/18/guatemala-ataques-la-libertad-de-prensa], letzter Aufruf 08.09.2021.

HRW (2021b): Argentina: Formosa adopta medidas abusivas en su respuesta al Covid-19. [https://www.hrw.org/es/news/2021/03/26/argentina-formosa-adopta-med idas-abusivas-en-su-respuesta-al-covid-19], letzter Aufruf 08.09.2021.

IDEA (2021): Grupo de expertas y expertos electorales: elecciones y Covid-19. [https://w ww.idea.int/es/news-media/news/es/grupo-de-expertas-y-expertos-electorales-elec ciones-y-covid-19], letzter Aufruf 08.09.2021.

Infobae (2020): Los respiradores chinos que llegaron a Bolivia para combatir el coronavirus no funcionan bien y hay denuncias de corrupción. [https://www.infobae.com/am erica/america-latina/2020/08/27/los-respiradores-chinos-que-llegaron-a-bolivia-pa ra-combatir-el-coronavirus-no-funcionan-bien-y-hay-denuncias-de-corrupcion/], letzter Aufruf 08.09.2021.

Lang, Kai-Olaf; Zilla, Claudia (2020): Kein Aufwind für populistisches Regieren in Corona-Zeiten. in: Lippert, Barbara; Mair, Stefan; Perthes, Volker (Hg.): Internationale Politik unter Pandemie-Bedingungen: Tendenzen und Perspektiven für 2021. SWP-Studie 2020/S 26, Dezember 2020, 20-23. [https://www.swp-berlin.o $\mathrm{rg} /$ publikation/internationale-politik-unter-pandemie-bedingungen/\#hd-d36438 e772], letzter Aufruf 08.09.2021.

Listin Diario (2021): Latinoamérica revive una vieja pandemia en medio del covid: la corrupción. [https://listindiario.com/la-republica/2021/03/07/660042/latinoamer ica-revive-una-vieja-pandemia-en-medio-del-covid-la-corrupcion], letzter Aufruf 08.09.2021. 
Meyer, Thomas (2006): Praxis der sozialen Demokratie, Wiesbaden: VS Verlag für Sozialwissenschaften.

Meyer, Thomas (2011): Theorie der sozialen Demokratie, Wiesbaden: VS Verlag für Sozialwissenschaften.

NODAL (2021): Más de 20 ministros y ministras de salud dejaron sus cargos desde inicios de la pandemia. [https:/www.nodal.am/2021/03/mas-de-20-ministros-y -ministras-de-salud-dejaron-sus-cargos-desde-el-inicio-de-la-pandemia/], letzter Aufruf 08.09.2021.

Rueschemeyer, Dietrich (2011): Political Equality, in: Badie, Bertrand; BergSchlosser, Dirk; Morlino, Leonardo (Hg.): International Encyclopedia of Political Science, Bd. 1. Thousand Oaks. CA: SAGE Publications. 818-823.

Schmidt, Manfred G. (2004): Korruption. Wörterbuch zur Politik. Stuttgart: Kröner Verlag.

TNYT (2020): Los villanos del virus en Latinoamérica: especuladores de equipos de protección y políticos corruptos, 10.08.2020. [https:/www.nytimes.com/es/2020/06 /20/espanol/america-latina/corrupcion-coronavirus-latinoamerica.html], letzter Aufruf 26.06.2021.

Urbinati, Nadia; Warren, Mark E. (2008): The Concept of Representation in Contemporary Theory, in: The Annual Review of Political Science 2008. 11, 387-412.

Urbinati, Nadia (2012): Competing for Liberty: The Republican Critique to Democracy., in: American Political Science Review. Vol. 106, No. 3, August, 607621.

Wesche, Philipp; Zilla, Claudia (2017): Korruption in Brasilien: Ein Fass ohne Boden: Der Lava-Jato-Fall, seine Aufklärung und die regionalen Implikationen, in: SWP-Aktuell 2017/A 39, Juni 2017. [https://www.swp-berlin.org/publications/ products/aktuell/2017A39_wse_zll.pdf], letzter Aufruf 08.09.2021.

Zilla, Claudia (2020a): Wissenschaftliche Politikberatung in Zeiten der Pandemie: Herausforderungen für die qualitativ-empirische Sozialforschung. Forschungsgruppe Amerika. Arbeitspapier Nr. 01, Dezember 2020. [https://www.swp-berlin.org/pu blications/products/arbeitspapiere/2020_AP_Zilla_Wiss_Politikberatung_Pande mie.pdf], letzter Aufruf 08.09.2021.

Zilla, Claudia (2020b): Corona Crisis and Political Confrontation in Brazil. The President, the People, and Democracy under Pressure, in: SWP Comment 2020/C 36, Juli 2020. [https:/www.swp-berlin.org/publications/products/aktuell/2020A 53_corona_brasilien.pdf], letzter Aufruf 08.09.2021.

Zilla, Claudia (2020c): Corona como metáfora, in: Agenda Pública. El Pais vom 04.09.2020 [https://agendapublica.es/corona-como-metafora/], letzter Aufruf 08.09.2021.

Zilla, Claudia; Keseberg, David (2019): Venezuela: Menschenrechte im Ausnahmezustand, in: Zeitschrift für Menschenrechte - Journal for Human Rights, Jg. 13, 2019, Nr. 2. Frankfurt a.M.: Wochenschau Verlag, 38-56. 
